to appear in Linguistic Variation Yearbook 2005

\title{
THE UNIVERSAL FORCE OF FREE CHOICE ANY ${ }^{1}$
}

\author{
VENEETA DAYAL \\ Rutgers University
}

\begin{abstract}
This paper adds to the ongoing debate about the quantificational status of English FC any. It argues that any is a universal, though its universality is such that it can be conjoined with an existential statement. This is established by showing, first of all, that various properties that are amenable to a universal account of FC any remain elusive in recent accounts that treat it as an indefinite. Secondly, it presents a detailed description of supplementary/numeral any, cases in which any occurs with an indefinite. Such cases have been assumed to provide evidence for the indefinite-any view but an explicit semantics for the constructions is given, showing that any invariably contributes universal quantificational force. Existential force always comes from other elements. The paper also discusses alternative formulations that preserve the key insights of the universalist position and the role of cross-linguistic variation in the study of FC items.
\end{abstract}

Keywords: Free choice items, Universal-any, Indefinite-any, Supplementary any, Numeral any, Cross-linguistic variation, Modality.

\section{Introduction}

This paper is concerned with the quantificational force of Free Choice (FC) any. In the first studies of this item, the question was posed as a binary choice between two possibilities, any as a universal or any as an existential quantifier. The discussion since then has become more nuanced, with attention being drawn not only to the quantificational force of any but also to its quantificational domain. Furthermore, our understanding of the quantificational variability of indefinites has added a layer of sophistication to the discussion. Still, the issue of any as a universal or any as an indefinite/existential continues to be debated. I will argue in this paper that FC any is indeed a universal but I will draw a distinction between two possible ways in which this universality 
contributes to meaning. One possibility is that it functions as a full-blown universal, the other that it supplies a condition of universality to an existential statement. The first we might take to be a form of direct universality, the second a form of indirect universality. I will show that a full account of FC any must allow for both types of universal quantificational force.

Since the word any has been subjected to semantic scrutiny for such a long time, perhaps I should provide some justification for yet another paper on the topic, specifically one dealing with a question that has plagued the study of this word since the very beginning. The primary justification lies in the fact that Free Choice any has, in fact, been somewhat neglected in favor of Negative Polarity any. While initial studies did focus on FC any, NPI any soon took center stage. From the eighties to the mid-nineties when FC any was discussed at all, it was usually as an addendum to NPI any (see, for example, Kadmon and Landman 1993). It was only recently that FC items began to be studied in their own right. Beginning with English any (Dayal 1995a, 1998) our understanding of FC items in English as well as other languages has been greatly enlarged (Quer 1998; Giannakidou 2001; Saeboe 2001; Kratzer and Shimoyama 2002; Zepter 2003; Chierchia 2004; Jayez and Tovena to appear, among others). This pattern of development has had some interesting consequences.

The first consequence of the inattention to FC any is that even after thirty years or more of semantic investigations there are lacunae in our knowledge of its empirical properties. FC items, for example, are known to be sensitive to the presence of modals but there is still little knowledge about their precise interactions. Furthermore, there are types of FC any that remain poorly understood and under-analyzed. This paper hopes to shed light on one such case, namely supplemental any, which has been brought into the literature by Jennings (1994) and Horn (2001, 2003). By adding to our understanding of the empirical properties of supplemental any, and its twin numeral any, the paper hopes to increase the descriptive base for future studies of FC items.

On the other side of the equation, the fact that focused studies of FC items have been conducted in the last ten years or so has been beneficial in at least one respect. Since cross-linguistic considerations play a far more central role in semantic argumentation today, recent work on FC any has been informed by insights based on data from a wide range of languages: Catalan, French, German, Greek, Italian, to name just a few. It is clear from these studies that the concept of FC is relevant in the grammar of natural languages, but the precise way in which FC is realized in different languages remains open, raising interesting questions regarding the nature of semantic variation. Any headway we make in our understanding of the English FC item any, therefore, also bears on our understanding of possible semantic variation in natural language. 
In the next section I will summarize the current debate on the quantificational force of any. I will draw on Dayal (1998) for universal-any and on Horn $(2001,2003)$ and Giannakidou (2001) as representatives of the indefinite-any view. In section 3 I will turn to the case of supplementary and numeral any, which have been presented as evidence against the universality of FC any. I will present these constructions in some detail since some of their properties have not been previously described. I will show that although these constructions cannot be handled in terms of direct universality, they are compatible with an indirectly universal approach. I will then return to an assessment of the universal-indefinite debate in light of the analysis of supplementary/universal-any and show that their properties remain elusive in the Horn-Giannakidou approach. I will also show that a universal-any account is not necessarily tied to a treatment of it as a universal quantifier. In doing so, I will draw a distinction between the indefinite-any approaches discussed in section 2 and non-quantificational universal-any accounts. I will end the paper with a brief discussion of the nature of possible variation with respect to FC items. I should clarify that although the paper mentions several recent accounts of FC items in English and other languages, detailed discussion of particular analyses is restricted to the authors mentioned in connection with section 2 . This is partly for reasons of space and partly to keep the discussion focused on the central theme of this paper, which is an analysis of supplemental/numeral any and the claim that FC any is always universal, whether direct or indirect.

\section{The universal-indefinite debate}

\subsection{Any as universal}

In discussing the meaning of any noun phrase the key question is determining its quantificational force, for which intuitive truth conditions can provide a reliable guide. In the case of any too, the standard practice of examining intuitive truth conditions is useful but there are other complicating factors, namely the issue of restricted distribution and nuanced interpretations. Recent accounts of FC any meet this challenge by looking at quantificational force, quantificational domain, as well as overarching distributional constraints. I will take as my starting point the position in Dayal (1998) on these three counts. There I demonstrated that the universal quantificational force of any cannot be imputed to the operators that yield generic indefinites, as claimed in Kadmon and Landman (1993), but must come from within. I implemented this by treating any as a universal quantifier. The difference between any and an ordinary universal came from their differing domains of quantification. Any quantified over the widest possible domain consistent with its property denoting argument, every quantified over the more familiar (extensional) 
domain. Finally, a requirement of vagueness was argued to be necessary to explain the full distribution of FC any. I will briefly list three phenomena that were crucial in motivating these features of the theory. Although they were used to argue against the proposal in Kadmon and Landman (1993), we will see that even later proponents of the indefinite-any view have not satisfactorily met the challenge posed by them. As such, they continue to be relevant in the debate about the quantificational force of any.

Let us begin with the argument from adverbs of quantification. In (1) and (2) we see that statements with a regular indefinite are compatible with adverbs of quantification but this is not true of statements with any. The adverb in (1b), like the one in (1c), only has a frequency reading. (2b)-(2c) end up being ungrammatical because they have i-level predicates that are not compatible with this use of the adverb. ${ }^{2}$ The conclusion that these data lead to is that FC any cannot be equated with a generic indefinite. Its universal force must be recognized as coming from within. Treating it as a universal quantifier is one way of doing that:

(1) a. An owl usually hunts mice.

b. Any owl usually hunts mice.

c. Every owl usually hunts mice.

(2) a. A lion is usually majestic.

b. *Any lion is usually majestic.

c. *Every lion is usually majestic.

The second phenomenon that motivated the analysis under discussion was subtrigging, first analyzed in LeGrand (1975). It presents the mirror image argument to the above, demonstrating an instance where the any phrase has universal force while the indefinite does not. This is shown by the paradigm in (3):

(3) a. John talked to a student who came up to him.

( $\forall$ reading unavailable)

b. John talked to any student who came up to him. ( $\forall$ reading available)

c. John talked to every student who came up to him. ( $\forall$ reading available)

Subtrigging was also a crucial factor in the conception of the domain of quantification as involving the widest possible set of individuals consistent with the descriptive content. The relevant contrast, fairly well-known by now, is between unmodified and modified any phrases in episodic statements: 
(4) a. *John read [any book].

b. John read [any book [he found]].

(5) a. $\quad \forall \mathrm{sx}[\operatorname{book}(\mathrm{x}, \mathrm{s})]\left[\mathrm{PAST}_{\mathrm{s} @}(\mathrm{~s}) \& \operatorname{read}(\mathrm{j}, \mathrm{x}, \mathrm{s})\right]$

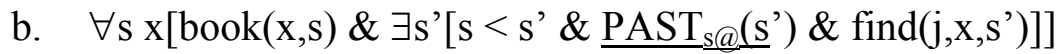
$\left[\operatorname{PAST}_{\mathrm{s} @}(\mathrm{~s}) \& \operatorname{read}(\mathrm{j}, \mathrm{x}, \mathrm{s})\right]$

The key idea in the explanation for the unacceptable (4a) is that quantifying over the widest possible domain results in presupposition failure. One cannot choose a domain that includes possible individuals and predicate something that is purely episodic of those individuals. ${ }^{3}$ The role of modification in repairing the presupposition failure in (4b) is shown in (5b). The temporal bound introduced by the relative clause restricts the domain appropriately. That this is a property of post-nominal modifiers in general is shown by the data in (6) where prepositional phrases and temporal locatives seem to have the same effect. (7a)-(7b) show that pre-nominal modifiers do not license any in the same way. However, (8a)-(8b), attributed to Barbara Partee and Jason Stanley respectively, show that given appropriate contexts a non-modified any may be interpreted as having some relevant covert restriction and become acceptable:

(6) a. John read any book on the table.

b. John talked to any student there.

(7) a. *John read any good book.

b. *John talked to any powerful politician.

(8) a. Mary confidently answered any objections.

b. After the dinner, we threw away any leftovers.

What is crucial for any, it appears, is the absence or presence of a modifier that introduces a temporal/spatial location, allowing the quantificational set to be narrowed down enough to avoid a presupposition clash. Postnominal modifiers, which are phrasal according to Sadler and Arnold (1994), provide such specification and are therefore sufficient for redeeming FC any in the absence of modality. Prenominal modifiers, which are lexical, do not introduce such specification. The proposed account explains why postnominal modifiers are sufficient to license any in episodic statements. The fact that other elements may introduce the crucial restriction covertly is not incompatible with this view. What seems to be lacking, at this point, is a good understanding of how and when such covert elements become available. However, we do know how to interpret them when and if they make their presence felt. 
The third phenomenon that was crucial in the account in Dayal (1998) had to do with the interaction of modals and partitive any. The fact that any phrases occur in partitives at all provide compelling evidence against the notion of widening as presented in Kadmon and Landman (1993) since the definiteness of the inner noun phrase necessarily limits interpretation to a contextually specified set of individuals. Sentences with partitive any phrases require that distributional constraints refer not just to the quantificational domain of any but also to the whole proposition. The formulation of this constraint is given in (9) below and the contrast it is supposed to capture in (10):

Vagueness Requirement: Any (A) (Op B) is felicitous iff $A \cap B$ is not contextually salient in any relevant world; where Op may be $\square, \diamond$, ! (permission), i (command) or null.

(10) a You may take any of these apples.

b. *You must take any of these apples.

This vagueness requirement has been criticized in the recent literature, not only by those who do not accept the universality of any but also by those who agree with it. Saeboe (2001: 769) finds it "debatable whether it offers precise predictions" and argues that "it is intrinsically non-compositional and not very explanatory". Chierchia (2004) also takes issue with it, "While the intuition behind domain vagueness might be sound, problems of implementation remain. It isn't clear, for example, how exactly to build domain vagueness into the lexical entry for any". And also, "Why do we need, in the end, two independent assumptions on the semantics of FC any: (i) that it is a modalized universal and (ii) that it is domain vague?" I will briefly demonstrate how this principle predicts the contrast noted above and show why we need to refer to a wide domain as well as vagueness at the level of the proposition in an account of FC any.

Consider the following logical representations of (10a) and (10b), respectively:

(11) a. $\forall \mathrm{x}[$ of-these-apples(x)] $\diamond[\operatorname{take}(\mathrm{you}, \mathrm{x})]$

b.

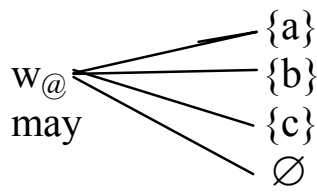


c. $\quad \Rightarrow$ A (possibly empty) subset of the set of apples will be taken in the actual world.

a. $\forall \mathrm{x}[$ of-these-apples(x)] $\square[\operatorname{take}($ you, $\mathrm{x})]$

b.

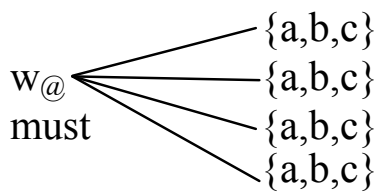

c. $=>$ Every apple in the set of apples will be taken in the actual world.

What comes into play is the fact that in the case of permissions, we cannot predict which apples, if any, will be picked in the actual world after the permission has been followed. In the case of commands, universal quantifycation over worlds forces each apple to be taken in each world, returning the full set of apples in the actual world. Partitives reveal the need for vagueness at the level of the proposition particularly clearly since the definiteness of the inner noun phrase does not allow vagueness to be satisfied via widening.

It is worth emphasizing that necessity modals are not inherently antithetical to vagueness. Compare the following sentences which differ only on whether they have a partitive any or a regular any. Regular any can occur with necessity, when subtrigged appropriately, if the set of entities is not contextually fixed. Partitive any, obviously, does not allow vagueness to be satisfied in this manner:

(13) a. You must eat any apple you bought.

b. *You must eat any of the apples you bought yesterday.

$$
\begin{aligned}
& \text { a. } \quad \forall \mathrm{x}[\text { poss-apple }(\mathrm{x}) \& \mathbf{P} \text { buy }(\mathrm{you}, \mathrm{x})]] \square[\operatorname{eat}(\mathrm{you}, \mathrm{x})] \\
& \text { a'. } \quad=>\text { Every member of an undetermined/potentially unknown set } \\
& \text { will be eaten } \\
& \text { b. } \quad \forall \mathrm{x}[\mathrm{x} \leq \text { the }(\text { apple } \& \lambda \mathrm{y} \mathbf{P}(\text { buy }(\mathrm{you}, \mathrm{y}))]] \square[\operatorname{eat}(\mathrm{you}, \mathrm{x})] \\
& \text { b'. } \quad=>\text { Every member of a determined and known set will be eaten }
\end{aligned}
$$

To make it even clearer that vagueness at the propositional level as well as modality in the quantificational domain is needed we can look at the following cases of ordinary any: 
(15) a. *John ate any apple.

b. *John must eat any apple that is lying here in front of us.

(15a) is ruled out because of the presupposition clash between too wide a domain of quantification and a narrow predication domain. Notably, vagueness is not violated. ( $15 \mathrm{~b})$ is ruled out as a violation of vagueness because the relative clause narrows down the set of apples to a contextually salient set. Crucially, there is no clash in presuppositions. ${ }^{4}$

Having outlined the key aspects of the direct universal approach, I will now turn to a discussion of the indefinite-any approach.

\subsection{Any as Indefinite}

A substantial amount of work on FC has come out since 1998. The most notable representatives of the view that any is an indefinite are Horn (2001, 2003) and Giannakidou (2001). ${ }^{5}$ Although their particular proposals are not identical, I take the cue from Horn himself in discussing them together. He notes that his description of the semantic properties of any as indefinite have not been formalized but the semantics in Giannakidou (2001) would be in keeping with his insights.

Briefly, Horn considers any to be an indiscriminative indefinite, like the FC item wh-ever in free relatives. Their contribution to meaning can be most easily conveyed by the locution 'it doesn't matter which'. The key ingredients of Giannakidou's account of FC any resonates with Horn's view of any as indiscrimative indefinite but differs in details:

(16) a. Condition Regulating the Distribution for any (Giannakidou 2001: 723)

(i) Any will not be grammatical in a sentence $\mathrm{S}$ if any is interpreted in the scope of a veridical expression in S.

(ii) In certain cases, clause (i) can be voided if $\mathrm{S}$ gives rise to a negative implicature.

b. Free Choice Item

(Giannakidou 2001: 706-707)

Let $\mathrm{W}_{\mathrm{i}}$ be a non-empty set of possible worlds. A sentence with a free choice item [[OP $\left.\left.\operatorname{DET}_{\mathrm{FC}}(\mathrm{P}, \mathrm{Q})\right]\right]$ is true in $\mathrm{W}_{0}$ with respect to $\mathrm{W}_{\mathrm{i}}$ iff:

(OP a non-veridical operator; $P$ the descriptive content of the of the FC-phrase; $Q$ the nucleus of the tripartite structure; $w_{0}$ the actual world) 
(i) Presupposition: $\forall \mathrm{w}_{1}, \mathrm{w}_{2} \in \mathrm{W}_{\mathrm{i}}$ : $\llbracket \alpha \rrbracket^{\mathrm{w} 1} \neq \llbracket \alpha \rrbracket^{\mathrm{w} 2}$ where $\alpha$ is the Free Choice phrase

(ii) Assertion: $\llbracket \mathrm{OP}_{\mathrm{w}, \mathrm{x}}[\mathrm{P}(\mathrm{x}, \mathrm{w}) ; \mathrm{Q}(\mathrm{x}, \mathrm{w})] \rrbracket=1$ where $\mathrm{x}, \mathrm{w}$ are the variables contributed by $\alpha$.

Giannakidou's account of the distributional restrictions on any is part of her general view of polarity licensing. For a list of the relevant (non)veridical operators I refer the reader to her papers (Giannakidou 1999, 2001), noting simply the importance it has in her account. The second part of her analysis concerns the semantics of FC items. She adopts the notion of i-alternatives, proposed by Dayal (1997) to account for wh-ever free relatives, in determining the set of worlds within which an any statement is to be evaluated. Its basic contribution is to require consideration of all possible values for the variable contributed by the any phrase. The any phrase itself is a Heimian indefinite having no quantificational force. It is bound by the non-veridical operator whose scope it occurs in. One final point to note is that the any phrase differs from an ordinary indefinite in contributing not only an individual variable but also a world variable. Though this particular feature will not play much of a role in our discussions I include it here for completeness. With this much in place we can turn to a substantive discussion of the indefinite-any approach.

Horn and Giannakidou make two kinds of arguments against the universalist approach. One, they present evidence to undercut some of the typical diagnostics used to classify universals, namely modifiers like almost, absolutely and exceptive phrases. Two, they draw attention to data that appear problematic for the universalist position. I will not have much to say about the criticism against the diagnostics except to note, as Horn and Giannakidou also do, that they do not provide evidence against the view that any is a universal. Rather, they show that the diagnostics do not separate out universal terms to the exclusion of other items and therefore no firm conclusion about the quantificational force of any can be based on them. As for the data they present in their papers as a challenge for the universalist position, one of these constructions will be dealt with at length in section 3. In this section I will instead use the three phenomena described in section 2.1 to assess the extent to which the indefinite-any approach presents a viable alternative to the universal-any approach.

Let us begin with the data in (1) and (2), showing the differential behavior of any vs. regular indefinites with respect to adverbs of quantification. Horn is silent on this point but Giannakidou (2001: 715-717) does take note of it and offers the following solution for the problem: ${ }^{6}$ 
(17) a. Any Yalie usually studies Homer.

b. (15a) is true in $w_{0}$ iff:

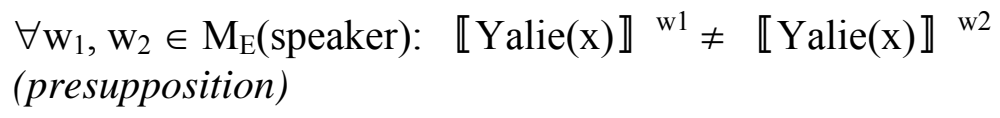

c. Most Yalies, whoever they might be, study Homer.

According to her, even though the any variable is bound by the operator USUALLY, the effect is nullified due to the presupposition of any: " $\mathrm{i}$ alternatives are drawn from the speaker's epistemic model, which includes the worlds the Q-operator ranges over, while the any/FC variable is bound by the Q-operator. This allows us to consider all values for any Yalie but actually assert a generalization about most of Yalies". 7 This is not very compelling as an explanation. For example, it is not clear that (17b) could not lead to a meaning similar to $(17 \mathrm{c})$. Putting this aside, this proposal makes certain predictions that are problematic for her account. ${ }^{8}$

One, if the presupposition of variation can make the Q-operator semantically ineffectual in this case, it is predicted that the presupposition will override all operators and the quantificational force of any will always be universal. This is not the intended consequence of the proposal but it is one that follows inevitably from the solution given for (17a). Consider the sentence in (18a), which has an existential interpretation, and forms one of the arguments used by Giannakidou against a universalist account of FC any. Applying the same logic as Giannakidou proposes for (17a), however, would result in a universal interpretation for (18a), regardless of the quantificational force of the imperative operator:

a. Pick any card

b. (18a) is true in $w_{0}$ iff:

$$
\begin{aligned}
& \forall \mathrm{w}_{1}, \mathrm{w}_{2} \in \mathrm{M}_{\mathrm{E}}(\text { speaker}): \llbracket \operatorname{card}(\mathrm{x}) \rrbracket^{\mathrm{w} 1} \neq \llbracket \operatorname{card}(\mathrm{x}) \rrbracket^{\mathrm{w} 2} \\
& \text { (presupposition) } \\
& \text { IMPERATIVE-OP s,x }[\operatorname{card}(\mathrm{x}, \mathrm{s}) ; \operatorname{pick}(\mathrm{you}, \mathrm{x}, \mathrm{s})] \\
& \text { (assertion) }
\end{aligned}
$$

We will further discuss the significance of such cases in section 4, noting simply the internal inconsistency in Giannakidou's account for now. 
The second problem posed by the proposed solution has to do with the phenomenon of Q-adverbs itself. We know that adverbs either function as quantificational operators or as frequency adverbs, but not both simultaneously. Now, the intuition about (17a) is not merely that we interpret any as a universal but also that we interpret the adverb as having a frequency reading. This could not be if the adverb were functioning as a Q-operator, even if that Q-operator has been rendered ineffectual by the presupposition of any. In fact, this can be established independently of intutions about (17a). If adverbs could bind any phrases in the manner shown in (17b), there would be no reason for any and adverbs to co-occur only with stage-level predicates. Giannakidou predicts their compatibility also with i-level predicates, contrary to what we know from examples like (2). ${ }^{9}$ Quite clearly, then, the problem regarding adverbs of quantification remains unexplained in Giannakidou's account of any.

Let us now turn to the second empirical phenomenon noted in Dayal (1998) as a problem for Kadmon and Landman's proposal of any as a Heimian indefinite, namely the role of subtrigging in licensing any in episodic statements. Giannakidou follows a long tradition, going back to LeGrand (1975), of treating subtrigged any sentences as involving hidden conditionals. While such a move seems plausible enough on the basis of intuitive truth conditions, there are several outstanding questions that a conditional analysis raises:

(19) a. John talked to any woman who came up to him

b. $\forall \mathrm{w}, \mathrm{x}[[\operatorname{woman}(\mathrm{x}, \mathrm{w}) \&$ came-up-to(x,j,w)] $\rightarrow$ talked-to(j,x,w)]

Note that the final logical representation that Giannakidou assigns subtrigged sentences is virtually the same as in Dayal (1998). Therefore, the issue to be resolved does not concern the final truth conditions of a subtrigged sentence but the motivations for arriving at the relevant representation and the explanation for the difference between a subtrigged and a non-subtrigged example. According to Giannakidou, the relative clause provides the restriction on the conditional while the universal operator in an episodic sentence, which is the construction of relevance here, comes from the iterativity of the predicates, especially the matrix. We will take up the various elements in this explanation in turn.

First of all, if the operator can come from the iterativity of the matrix predicate, there is no principled explanation for the obligatoriness of the relative clause. The head noun should be able to provide the restriction. After all, the literature on bare plurals and generic indefinites, on which the indefinite-any view is based, is replete with cases where the head noun provides the crucial restriction on the quantifier. (20a) should not differ from 
(20c) with respect to the ability of an unmodified common noun to provide the restrictive phrase in the quantificational structure:

(20) a. *John talked to any woman.

b. $\forall \mathrm{w}, \mathrm{x}[\operatorname{woman}(\mathrm{x}, \mathrm{w}) \rightarrow$ talked-to $(\mathrm{j}, \mathrm{x}, \mathrm{w})]$

c. Cows eat grass. / A cow eats grass.

d. $\forall \mathrm{w}, \mathrm{x}[\operatorname{cow}(\mathrm{x}, \mathrm{w}) \rightarrow \exists \mathrm{y}[\operatorname{grass}(\mathrm{y}) \& \operatorname{eat}(\mathrm{x}, \mathrm{y}, \mathrm{w})]]$

A second problem arises due to the fact that Giannakidou's explanation for subtrigging does not make any reference to the presupposition about variability, the only difference between FC any and an indefinite in her account. ${ }^{10}$ It stands to reason, therefore, that if an any sentence can have a conditional structure, so should the corresponding sentence with a regular indefinite. But we can see from (21a) that such sentences do not have universal interpretations. There is a similar problem for sentences with partitive any, such as (21b), which remain ungrammatical in spite of subtrigging. One would like to know why the conditional structure does not come into play and redeem the sentence. Note that the definiteness of the inner noun phrase cannot be held responsible for its unacceptability since that definiteness is completely innocuous in any statements with possibility modals, where also such binding would presumably be needed:

(21) a. John talked to a woman who came up to him.

b. *John talked to any of the women who came up to him.

There are two further points that are worth drawing attention to here. Giannakidou's discussion regarding the conditional analysis of subtrigging is based on an incomplete paradigm. In Dayal (1995a, 1998) I noted that iterativity is not a necessary condition for subtrigging, though it may be sufficient (see also Jayez and Tovena to appear). This conclusion was based on cases like (22a). I add (22b), which uses the kind of predicate Giannakidou claims cannot provide the operator for the conditional structure because it is non-iterative. Both sentences are fully acceptable with non-iterative interpretations. All that is required is that the set of individuals who satisfy the conditions in the any phrase not be contextually determined, as predicted by the vagueness requirement: ${ }^{11}$

(22) a. At the end of his speech, the President thanked any soldier who had fought in the Gulf War.

b. Anybody who was there at that time died in the blast. 
Let me finally entertain the possibility that the inherent plurality of the any phrase, due to its presupposition of variation, may be responsible for the reanalysis of a subtrigged sentence as a conditional. There is, perhaps, some indirect evidence for this in that the bare plural version of (21a) is able to get universal readings. Let us set aside the issue of why plurality should have this effect and see whether the idea itself can be maintained. The primary problem I see with taking this line is that, again, there is no reason for the obligatoriness of the relative clause. If plurality can trigger a conditional analysis and the plurality comes from the presupposition about i-alternatives, the head noun by itself should be sufficient to redeem any.

We have seen, then, that once we subject the conditional analysis of subtrigging to scrutiny various stipulations become necessary to capture the facts and the idea loses much of its initial appeal. Unless all of the features of the explanation can be motivated and justified, the claim that subtrigging involves a conditional structure remains a description of the intuitions that a theory of any needs to capture, not an explanation of the problem.

Let us now look at the third phenomenon, having to do with the interaction of modality and partitive any, that was central to the universal-any proposal of Dayal (1998). Recall the key facts:

a. You may take any of these apples.

b. *You must take any of these apples.

Neither Horn nor Giannakidou attempt an explanation but it is easily demonstrated that no ready solution for the paradigm exists in the indefiniteany approach. A reviewer sympathetic to the indefinitist position suggests that the reason for the unacceptability of $(23 \mathrm{~b})$ is that the definite inside the any phrase does not provide the situation variable that Giannakidou's account requires. As pointed out in connection with the subtrigging cases, this idea is obviously unworkable. The culprit for the ungrammaticality of (23b) cannot be the definiteness of the inner NP since it causes no problems with the possibility modal in (23a). Presumably, that too would require a situation variable for the operator to bind.

It may be worth noting here that accounts like Saeboe (2001), which posit universal force for any but do away with a constraint against indeterminacy at the propositional level, are also unable to handle the contrast at issue. ${ }^{12}$ Recall that the explanation in Dayal (1998) crucially depends on there being two quantifiers, one contributed by the modal, existential or universal as the case may be, and one contributed by FC any, a universal. The combination of two universals with the definiteness of the inner noun phrase is what leads to a violation of vagueness in the case of necessity. Thus, even if we have reservations about this constraint, we seem to have no alternative that 
we can use to handle the relevant contrast, at least at the present time. We can either choose to ignore this data and keep our theory pristine or we can keep the contrast in public view and take it as a challenge to improve upon the specific implementation of the constraint. The discussion in the following sections will show that keeping the partitive data in mind is important in advancing our understanding of the quantificational force of any. The task of improving on the implementation of the vagueness requirement I will leave for future research, pointing the reader to Jayez and Tovena (to appear) whose principle of non-individuation provides an alternative to vagueness. As they note, a compositional way of deriving these facts may not ultimately be possible.

In this section I have reviewed the universal-any position and showed that the arguments leveled against the indefinite-any position of Kadmon and Landman (1993) have not been addressed by the indefinite-any accounts in Horn (2001, 2003) and Giannakidou (2001). These recent studies, however, do raise some fresh challenges to the universal-any position that need be taken seriously.

\section{Supplementary and numeral free choice any}

\subsection{The facts}

In this section I turn to one of the constructions that has been brought up in the recent literature as evidence against the univeral-any view, namely supplementary any, a term due to Jennings (1994). ${ }^{13}$ These cases are also noted by Horn (2001, 2003), Giannakidou (2001) and Jayez and Tovena (to appear) as intractable for the universal-any account. Supplementary any is illustrated in (24a). I will also include numeral any, illustrated in (24b), in my discussion here since supplementary and numeral any have several properties in common.

(24) a. Take an apple, any apple.

b. Take any two apples.

The problem such examples pose for universal-any is actually foreshadowed by the observation in Vendler (1967:82), who notes that "Any leaves you free both as to which and how many to choose [in permissions]". He goes on to note, however, that "this indifference to size has a curious limitation: "if I formulate my offer in terms of any, there will be an upper limit to my generosity...the immediate scope of any cannot exhaust the total population; in other words, any never amounts to every." This can be demonstrated by the following example, where any numeral is acceptable as long as it is not identical to the total number of apples: 
(25) a. You may take any one/two/.../n-1 apple(s).

$n=$ total number of apples

b. *You may take any $\mathrm{n}$ apples.

c. $\quad \forall \mathrm{x}[[\operatorname{apple}(\mathrm{x}) \& \mathrm{~m}(\mathrm{x})] \rightarrow \operatorname{may}-\operatorname{take}(\mathrm{you}, \mathrm{x})]$

Note that this, in and of itself, does not argue against a treatment of FC any as universal since the upper limit could be derived by appealing to the need of a universal quantifier for a plural domain. If there are three apples, setting $\mathrm{m}$ at 1 or 2 will result in three possible assignments to $\mathrm{x}$ but setting $\mathrm{m}$ at 3 will necessarily yield only one possible assignment. However, there are other features of these constructions that need to be analyzed in order to see how they relate to the universal-any approach.

Although numeral/supplementary any are not entirely unknown in the literature, it would be fair to say that not many of their properties have been documented. I will therefore begin by laying out some of their core features. There are some respects in which supplementary/numeral any behave just like regular FC any. They are not acceptable in episodic statements (cf. (26a) and (27a)), but are compatible with permissions (cf. (26b) and (27b)), as well as with possibility modals (cf. (26c) and (27c)). As we can see, in structures where they are acceptable, we have the possibility of an ordinary any or a partitive any:

(26) a. *John chose an apple, any apple / any of these apples.

b. Choose an apple, any apple / any of these apples.

c. You may choose an apple, any apple / any of these apples.

a. *John chose any two apples / any two of these apples.

b. Choose any two apples / any two of these apples.

c. You may choose any two apples / any two of these apples.

However, the set of contexts that allow supplementary and numeral any is not identical to the set that allows regular FC any. Subtrigging, for example, is not a hospitable context for supplementary and numeral any and necessity modals license supplementary and numeral any even with partitives. As we can see below, the judgments for regular any, given in (28c) and (29c), are the opposite of the judgments for supplementary and numeral any, given in (28a-b) and (29a-b):

(28) a. *John chose an apple that was red, any apple that was red / any of the apples that were red.

b. *John chose any two (of the) apples that were red. 
c. John chose any apple that was red.

a. John must choose an apple, any apple / any (one) of these apples.

b. John must choose any two apples / any two of these apples.

c. *John must choose any apple / any of these apples.

What this tells us is that conclusions we might draw on the basis of numeral and supplementary any will not necessarily transfer over to regular any. This is further confirmed by considering opaque contexts. There are some non-modal intensional contexts that license supplementary any but regular any is not possible there. Compare (30), fashioned after examples in Jennings (1994), with (31):

(30) a. John is looking for an apple, any apple.

b. She was waiting for a policeman, any policeman, to show up.

(31) a. *John is looking for any apple.

b. *She was waiting for any policeman to show up.

It is also worth noting that in such cases, supplementary any is not comfortable in a partitive phrase:

(32) a. John is looking for an apple, (*any of these apples).

b. She was waiting for a policeman, (*any of the policemen), to show up.

Finally, not all opaque contexts seem to license supplementary any. Verbs like dream or imagine are not hospitable environments, as shown in (33). Note though that this is not a feature that separates supplementary any from regular FC any. The point of the example here is to distinguish two types of opaque contexts, one that can and one that cannot host supplementary any:

(33) a. *John dreamt of a movie star, any movie star.

b. *John imagined a unicorn, any unicorn.

Turning to numeral any in opaque contexts, they are acceptable, it seems, only with the aid of purpose clauses:

(34) a. ?John is looking for any two apples.

b. John is looking for any two people to play the lead parts.

c. John wants to talk to any two movie stars for his article. 
Before embarking on an analysis of these data, I would like to underscore the relevance of the differences between regular and supplementary/numeral any. It may well be possible to rule out some of the the unacceptable cases of supplementary any on the basis of (non)veridicality, using Giannakidou (1999, 2001), or with some additional conditions added to the indefinite-any account. The point, however, is that given the distinct distributional facts about supplementary/numeral any and regular FC any, a straightforwardly unified account is not possible. ${ }^{14}$ The proponents of the indefinite-any view have focused on the simple fact of co-occurence compatibility and glossed over the problem of distributional differences. They have taken semantic compatibility as evidence of semantic identity, a conclusion that it not warranted by the facts. We will return to a further discussion of these issues in section 4.1 after we have spelt out an analysis for supplementary/numeral any that is able to deal with these distributional distinctions.

\subsection{An analysis}

Starting with supplementary any, we can take possible elaborations of the supplementary clause as our guide in deciding on its semantics. On the basis of (35), then, we might offer (36) as a plausible representation of a supplementary any sentence: ${ }^{15}$

(35) a. You may choose an apple, it could be any apple / any apple could be the one you choose.

b. You must choose an apple, it could be any apple / any apple could be the one you choose.

Consider the possibility statement first:
a. $\diamond_{\mathrm{i}} \exists \mathrm{x}[\operatorname{apple}(\mathrm{x}) \& \operatorname{choose}(\mathrm{you}, \mathrm{x}) \&$
antecedent clause
$\left.\forall \mathrm{y}\left[\operatorname{apple}(\mathrm{y}) \rightarrow \diamond_{\mathrm{i}} \operatorname{choose}(\mathrm{you}, \mathrm{y})\right]\right]$ supplementary clause
b. $\diamond_{\mathrm{i}} \exists \mathrm{x}[\operatorname{apple}(\mathrm{x}) \& \operatorname{choose}(\mathrm{you}, \mathrm{x}) \&$ antecedent clause $\left.\left.\forall \mathrm{y}[\operatorname{apple}(\mathrm{y})) \rightarrow \diamond_{\mathrm{i}} \mathrm{x}[\operatorname{choose}(\mathrm{you}, \mathrm{x})]=\mathrm{y}\right]\right]$ supplementary clause

c.

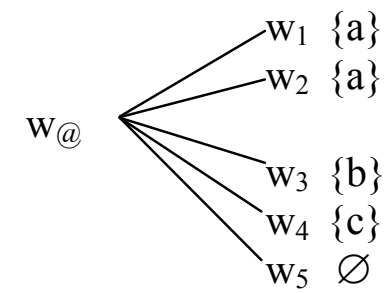


The antecedent clause, the statement without any, states that an apple will be chosen in at least one accessible world. The supplementary clause brings in universality as well as another possibility modal. This second modal, I should point out, is not postulated as part of the meaning of any but is rather motivated by the presence of an overt modal in the full supplementary clause, as seen in (35). The claim I would like to make is that semantically integrating the supplementary clause involves this second modal inheriting its base from the primary modal. This is represented above by coindexing the two modals. Assume there are three apples, the antecedent clause forces there to be at least one world in the modal base that contains an apple that is picked. The supplementary clause requires that for each apple, there be a world where that apple is the one picked. Vagueness is satisfied since it cannot be predicted which apple, if any, will be picked in the actual world. The case of numeralany can be treated in like fashion, except that there will be an overt restriction with respect to number.

Turning now to necessity, the only difference is that the antecedent clause forces every world in the modal base to contain an apple that is picked, due to the logic of necessity. The supplementary clause requires that for each apple, there be a world where it be picked, as in the case of possibility. Note that vagueness will still be satisfied since it still cannot be predicted which apple will be picked in the actual world. This is shown diagrammatically in $(37 \mathrm{c})$ :

$$
\begin{array}{lll}
\text { a. } & \square_{\mathrm{i}} \exists \mathrm{x}[\operatorname{apple}(\mathrm{x}) \& \operatorname{choose}(\mathrm{you}, \mathrm{x}) \& & \text { antecedent clause } \\
& \left.\forall \mathrm{y}\left[\operatorname{apple}(\mathrm{y}) \rightarrow \diamond_{\mathrm{i}} \operatorname{choose}(\mathrm{you}, \mathrm{y})\right]\right] & \text { supplementary clause } \\
\text { b. } & \square_{\mathrm{i}} \exists \mathrm{x}[\operatorname{apple}(\mathrm{x}) \& \operatorname{choose}(\mathrm{you}, \mathrm{x}) \& & \text { antecedent clause } \\
& \forall \mathrm{y}\left[\operatorname{apple}(\mathrm{y}) \rightarrow \diamond_{\mathrm{i}} \mathrm{x}[\operatorname{choose}(\mathrm{you}, \mathrm{x})=\mathrm{y}]\right] & \text { supplementary clause }
\end{array}
$$

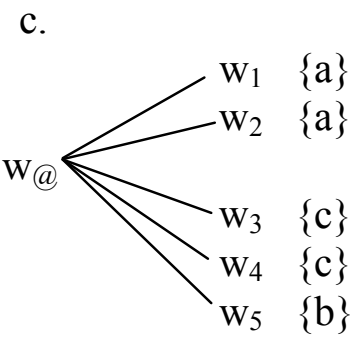

A few points are worth drawing attention to. The contribution of the supplementary clause, we can see, is the same in both cases. This raises a question about a difference that has been observed between possibility and necessity, having to do with the strictness of the number implicatures generated: 
(38) a. You may take two apples, any two.

(*You could, of course, take more).

b. You may take any two apples

(*though you could take more).

c. You must take two apples, any two.

(You could, of course, take more).

d. You must take any two apples

(though you could take more).

A permission to take one apple seems to entail that only one apple can be taken. The corresponding command, on the other hand, does not impose a similar restriction. It is worth noting that this difference is due to the logic of permission vs. command in general. Permission involves the lifting of a ban so that the conversational implicatures related to number are operative in the antecedent clause, which in the case of (38a)-(38b) is you may take only one apple. Commands do not presuppose a similar ban since they fix only a minimum requirement you must take at least one apple. (39) is an acceptable model for commands where there are worlds in which more than the specified number of apples are taken but a corresponding model for permissions would not be acceptable:

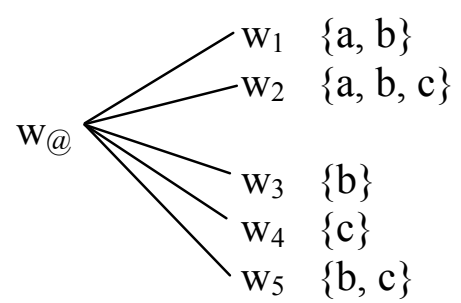

There is no reason, therefore, to introduce any distinction in the supplementary clause to capture the contrast in number implicatures between permissions and commands. Of course, there may be additional assumptions that might introduce number restrictions in commands as in You must/will marry any one doctor (Kratzer and Shimoyama 2002; Chierchia 2004), where cultural assumptions lead to restrictions on number. The point is that this is is not a general requirement in commands: ${ }^{16}$

(40) a. You must answer (any) two questions on this page but if you answer more you will not be penalized. 
b. To win this game you must have (any) four cards in a sequence but having more cards in a sequence will not disqualify you.

I have presented an account of supplementary and numeral any as having a hidden modal which draws on the same modal base as the modal in the antecedent clause. This has interesting consequences for episodic statements where no modal base is provided by the antecedent clause:

(41) a. *John chose any one apple.

b. *John chose an apple, any apple.

c. $\exists \mathrm{x}[\operatorname{apple}(\mathrm{x}) \& \operatorname{chose}(\mathrm{j}, \mathrm{x})] \& \forall \mathrm{y}[[\operatorname{apple}(\mathrm{y})] \rightarrow \diamond \operatorname{chose}(\mathrm{j}, \mathrm{y})]$

If (41a) or (41b) were grammatical, they would have representations like (41c). The problem with the representation, I would like to claim, is with the modal base for the supplementary clause. Since there is no modal in the antecedent clause to draw on, the only possibility would be for it to be cued to the speaker's knowledge about identity. And, in fact, there are FC items in English that convey precisely this type of modality, as shown below:

(42) a. John chose whichever apple Mary was pointing out to him, but I couldn't tell you which one.

b. John chose an apple, some apple or other. I couldn't tell you which one.

In Dayal (1997) FC readings of free relatives are analysed essentially along the lines being considered here. The free relative introduces a unique entity with the relevant property and the $\mathrm{FC}$ item ever introduces universal quantification over identity-alternatives for it. ${ }^{17}$ And although I have not seen any detailed discussion of some $N$ or other, it clearly represents the indefinite counterpart of such statements. The fact that numeral/supplementary any do not lend themselves to this interpretation, tells us that FC any does not encode the kind of epistemic modality that is at stake in these cases. This conclusion is in keeping with the distinction Vlachou (2003) makes between FC readings of free relatives and any statements and is at least at odds with the claims in Horn's and Giannakidou's work (see also Jayez and Tovena to appear on this). ${ }^{18}$

Note that we have not drawn on the impossibility of any in the episodic antecedent clause to rule out supplementary/numeral any in these cases. The reason, obviously, is that using the compatibility of the antecedent clause with FC any to explain its co-occurence with numeral/supplementary any cannot work as a general strategy. We have seen that there is no reliable correlation 
between the ability of an antecedent clause to host regular FC any and its ability to host numeral/supplementary any.

Finally, let us look at the behavior of supplementary/numeral any in opaque contexts. Recall that there were differences between opaque verbs with respect to hosting supplementary any and that purpose clauses were needed to license numeral any:

(43) a. John imagined / dreamt of a unicorn, *any unicorn.

b. John wanted/was looking for a unicorn, any unicorn.

(44) a. *John is looking for any two people.

b. John is looking for any two people to play the lead roles in the play.

The claim I would like to make is that verbs like want or look for but not dream or imagine have, as part of their lexical semantics, a purpose argument that the supplementary modal can draw on. ${ }^{19}$ The data below illustrates the kind of distinction I am alluding to here:

(45) a. John was looking for a policeman to report the crime to.

b. John wanted a book to read.

c. *John imagined a country to go to.

d. *John dreamt of a woman to talk to.

If this observation is on the right track, then the interpretation of supplementary/numeral any may include reference to the purpose clause argument. (43b), for example, would be interpreted in the following way, adopting here the analysis of opaque verbs as relating to properties (Zimmermann 1993):

(46) a. John is looking for a unicorn for some purpose and any unicorn could serve that purpose.

b. look-for $\left(\mathrm{j}, \lambda \mathrm{x}\right.$ unicorn $\left.(\mathrm{x}), \varphi_{\mathrm{i}}\right) \&$

$\forall \mathrm{y}\left[\right.$ unicorn $(\mathrm{y}) \rightarrow \diamond_{\mathrm{j}}$ 's search is successful fulfil $\left.\left(\mathrm{y}, \varphi_{\mathrm{i}}\right)\right]$

The possibility modal does not have to draw on identity-alternatives since the intensional context provides the necessary base. Furthermore, I am suggesting that a purpose clause comes into play in these cases, separating out those intensional verbs that support supplementary any and those that do not. The final remaining question is why an overt purpose clause is needed in the case of numeral any. This, I believe, might be because no generic reason can be imputed for a relation to $n$ (but not $n-1$ or $n+1$ ) entities of the relevant sort. 
In this section I have looked at a range of examples involving supplementary/numeral any and identified some properties that had not been previously noted in the literature. What we have seen in the supplementary any cases is that FC any is not at odds with indefiniteness, but no more than that. The conclusion drawn by the proponents of the indefinite-any view that supplementary any, and by extension all FC any, is not a universal was hasty. I have presented an explicit account of the contribution of any in these sentences as involving universal quantification in combination with covert non identityoriented modality. This, I have demonstrated, is compatible with existential statements introduced by indefinites. No doubt more needs to be done towards analyzing supplementary any but the headway we have already made provides us with a good basis for returning to the debate about the quantificational force of FC any.

\section{Back to the universal-indefinite debate}

\subsection{Supplementary/numeral any and the universal-any position}

Let us return to the question whether FC any is a universal or an indefinite, using insights gained from the analysis of supplementary/numeral any above. The conclusion we come to is that FC any is always a universal. However, its universality can be expressed either directly, as in the account of Dayal (1998) which was summarized in section 2.1, or it can be expressed indirectly such that the universality contributed by any is conjoined with an existential statement in the way elaborated in section 3.2. This, it is worth emphasizing, does not represent a case of ambiguity. Rather, direct universality is the core meaning of FC any, with indirect universality coming into the picture only when other elements contribute an existential quantificational bound. These claims are based on empirical considerations discussed in sections 2 and 3, which I repeat here:
a. *John must answer any of these questions.
b. John must answer any one question.
c. John must answer a question, any one of these questions.

We know how the universalist account uses the universality of any and necessity along with the definiteness of partitivity to derive the ungrammaticality of (47a). And we know that numeral and supplementary any can be accounted for by conjoining the universality of any with an existential statement provided by the numeral or the indefinite in the antecedent clause. (47a) shows that in the absence of an indefinite there is no possibility of an existential bound to the universality of any. Otherwise, it would be expected 
that (47a) would be acceptable under the indirect universal aspect of any. Thus, we take any to be a universal, but one that can augment an existential statement if, and only if, the existential statement is independently introduced. Consider, in this connection, imperatives like (48a) and (48b) discussed by Horn (2001, 2003), Giannakidou (2001) and Jayez and Tovena (to appear). What is interesting about these examples is that an indirect universal interpretation seems to be available without the presence of an indefinite. To counterbalance, we might add (48c) from Dayal (1998), attributed to Manfred Krifka, in which any is clearly functioning as a direct universal. Similarly, any plays a non-supplementary, directly universal role in (48d):

(48) a. Press any key.

b. Pick any card.

c. Confiscate any liquor.

d. Throw away any leftover food immediately.

The paradigm in (48) shows that the quantificational force of any can vary between existential and universal but it does not show that any is ambiguous. ${ }^{20}$ That is, we do not interpret these sentences as either pick/press/confiscate/ throw-away one or pick/press/confiscate/throw-away all. In each case the interpretation is fixed, pick/press one in the case of (48a)-(48b) and confiscate/ throw-away all in the caseof (48c)-(48d). Let us see how this can be explained.

Taking the existential statements in (48a)-(48b) first, we note that they are not interpreted in a neutral context. World knowledge tells us that particular keys on a computer keyboard have specialized functions. (48a) is therefore easily interpreted against a contextually supplied existential statement and FC any can play a supplementary role, providing the requisite universality. Similarly, picking a card is a well-known step in a card game or magic trick. Once again, the context gives rise to assumptions that entail an existential statement, allowing any to function as a supplementary clause. The elaborations in (49) show that such existentially interpreted imperatives typically go hand in hand with statements of purpose:

(49) a. To continue, press any key.

b. Pick any card. I will tell you what it is.

Turning now to (48c)-(48d), no such context comes to mind. The most likely scenario in which they could be uttered would be one where there is an expectation that contraband liquor can be found or that there is some unspecified amount of leftover food. That is, there has to be overt or covert subtrigging and the set of contraband items or leftover food not contextually 
fixed. Without an independently supplied existential statement, the quantification of any remains universal, as expected.

The data in (48)-(49), then, show that contextual priming may be enough to introduce an existential quantificational bound, nudging FC any to be indirectly universal. They further show that in the absence of such priming, the universality of FC any remains untouched. To conclude this section, we have seen that supplementary/numeral any do not require weakening of the claim that FC any is a universal. They simply show that the universality of any is compatible with an existential bound, if that bound is independently introduced.

\subsection{Supplementary/numeral any and the indefinite-any position}

We will now consider the indefinite-any proposal in light of what we have learnt about supplementary/numeral any. Let us begin by considering the essential ingredients of the indefinite-any view, given below in schematic form:

$$
\text { OP } x\left[\ldots x_{\text {any }}\{+ \text { variation/indiscriminacy }\} \ldots\right]
$$

Any contributes a variable just like an indefinite. It has an added feature distinguishing it from indefinites, namely a presupposition of variation or some other way of enforcing indiscriminacy. There are two sources from which any can derive its quantificational force. One is the generic operator that gives universal force to indefinites. The other potential source is the added feature of variation/indiscriminacy (cf. the discussion in section 2.2 regarding Giannakidou's implementation). One way of understanding the quantificational implications of this schema is the following. Binding by a generic operator results in universal quantificational force, analogous to the case of generic indefinites. Unlike indefinites, however, even when there is an existential binding the any phrase a kind of pseudo-universal flavor creeps in due to the added feature of variation/indiscriminacy. Let us now see to what extent this way of accounting for the properties of any deals with the data we have been concerned with in this paper.

We already noted in section 2 that generic binding of any phrases is problematic since adverbs of quantification are not able to do so. There is no principled reason why only a covert generic operator would be able to do what an overt operator cannot. Furthermore, it was noted that it is not possible to derive all cases of FC any by appealing to a generic operator since there are many contexts in which FC any is available but indefinites have existential readings. Let us take another look at the key examples and see what the indefinite-any theory predicts about them: 
(51) a. Pick (a card) any card. $\exists+$ Variation/Indiscriminacy

b. John talked to any woman who came up to him. $\quad \forall$

c. Confiscate any liquor

d. John must answer any question he is asked.

We can take the example in (51a) as representative of numeral/supplementary any. We know that there is no generic operator here so it has to be the added feature of variation/indiscriminacy that brings in the flavor of universality. Let us turn now to the sentences in (51b)-(51d). As discussed earlier in this paper, all of them involve subtrigging, either covert or overt. The point to note is that their quantificational force is purely universal. Recall that Giannakidou (2001) proposes a conditional structure for sentences like (51b), a move that I have already critiqued in section 2.2. If we put aside those objections for the sake of argument and grant the validity of that move, we can certainly derive the universal reading these sentences have. What is not clear, however, is why the sentences are not ambiguous between the universal reading that the conditional analysis yields and an existential plus variation/indiscriminacy reading, an indirect universal reading, such as we find in (51a). The absence of a nonconditional reading for (51b) could, perhaps, be explained by appealing to the notion of (non)veridicality to rule out the non-conditional structure but that obviously would not apply to (51c)-(51d). Imperatives and modals are not hostile environments for FC any. The only way of capturing the facts would be to say that not only is a conditional analysis of subtrigging possible, but strictly required. This is a move for which there is no independent motivation. Furthermore, if a conditional structure is made obligatory, it would predict that a subtrigged version of (51a), pick any card you want, would also have only a universal reading. This, obviously, is incorrect. The magic trick or card game is supposed to proceed with only one card being picked, whether subtrigging is there or not.

To conclude, we have seen the pitfalls of taking simple co-occurrence facts as evidence of semantic identity. It is, of course, possible that the approach could be developed to give it adequate empirical coverage but clearly that is a task that has not yet been undertaken. Our conclusion, therefore, is that the indefinite-any approach is unworkable. FC any is a universal, albeit one that can be conjoined with existential statements without leading to contradiction.

\subsection{Universality without universal quantifier}

I have argued here for the universality of FC any, representing it as a universal quantifier. In this section I would like to step back and show that treating any as a universal quantifier is only one way of capturing its universality. Other 
quantifier-free approaches are possible that do not compromise the essential insights of the universal-any approach.

In trying to arrive at some unification of FC any and NPI any, I made the following observation in Dayal (1998). Taking FC any to be universal and NPI any to be existential, I suggested that both any's are licit in sentences that entail a universal statement: Any $\varphi \psi$ iff $\exists x[\varphi(\mathrm{x}) \& \psi(\mathrm{x})]$ entails $\forall \mathrm{x}[\varphi(\mathrm{x}) \rightarrow$ $\psi(\mathrm{x})$ ] (see also Jackson 1995; Zepter 2003). Although the existential claim here is meant for NPI any, which occurs in downward entailing contexts, it is worth pointing out that the essential claim of that paper, that the universality of FC any cannot be imputed to the same operators that give indefinites universal force, would not be affected if we wanted to treat FC any as an existential. What would be needed, of course, would be a principled way of ensuring that universality could be derived in the relevant contexts.

Some clarification is called for since the proposal now under discussion looks surprisingly similar to the indefinite-any accounts I have been arguing against. It recalls, in particular, Giannakidou's explanation for the fact that FC any cannot be bound by adverbs of quantification where the presupposition of variation was held responsible for the quantificational force. Such proposals, I am suggesting, would qualify as a directly universal account if it incorporated two features. ${ }^{21}$ It should not treat FC any like a Heimian indefinite. That is, FC any should never gets its universal quantificational force from a generic operator. Its universal quantificational force should always come from elements of meaning associated with FC any itself. And, its universality must be invariant. FC any must not introduce the existential quantificational bound in the supplementary/numeral/imperative cases that we have been discussing. That existential bound should come from other elements in the structure, such as an overt indefinite or a contextually supplied existential statement. I will briefly mention a recent proposal that has these properties.

Chierchia (2004) notes that the essential insights of Dayal (1998) can be captured in a theory of FC any that does not treat it as a universal quantifier. Building on Kratzer and Shimoyama (2002), Chierchia develops an account in which widening in the domain of an existential quantifier can be seen as strengthening. ${ }^{22}$ Chierchia takes a FC item to be an indefinite over the widest possible domain. In addition, it comes with a lexically triggered set of alternatives over its domain. This set of alternatives is made up of all the smaller domains, namely the subsets of the FC domain. The choice of the widest domain in the assertion combined with the implicatures it gives rise to results in a de facto universal interpretation for the any statement. Let us see how this works with the help of an example, presented here in simplified form: 
(52) a. (Yesterday) I saw any student (that wanted to see me)

b. I saw some student

assertion

c. I saw some student $\mathrm{Di} \&$ I didn't see some student ${ }_{\mathrm{Dj}}$ alternatives

d. $\neg[50 \mathrm{c}]$

implicature

Chierchia outlines the following steps to show how universality comes about. The first step is that the speaker chose to utter (52a), whose basic meaning is $(52 \mathrm{~b})$, instead of one of its competitors with a narrower domain. It follows from the choice of the wider domain in the assertion that the speaker was not in a position to say/imply (52c), which is stronger and would have been relevant. Assuming the speaker to be well informed, this means that she believes that (52c) does not to hold (i.e. she believes (52d)). This last step, according to him, "is the familiar 'leap of faith' present in quantity implicature...from 'not believing' to 'believing not'." He points out that once all of this is put together, the effect is that of a universally quantified statement. Thus, he derives universality while treating FC any as an indefinite, using Gricean reasoning along with the notion of the widest domain. Crucially, he does not invoke generic binding of the any phrase. As far as the existential readings of FC items is concerned, Chierchia notes that it has to be triggered by overt indefinite morphology. This is partly based on the paradigm of FC items in German and Italian. The key insight here is that the scalar implicatures generated by the indefinite play a role in restricting the size of the domains. ${ }^{23}$ This captures the second key element of a universal-any approach, namely that the default interpretation of FC any is that of a universal, it is not able to introduce the existential bound by itself.

In the interest of space, I will not go any further into the details of this account nor compare it with the proposal argued for in this paper. ${ }^{24}$ The idea here is merely to present an alternative approach that starts with similar premises as the Horn-Giannakidou approach but does not make the same commitments they do to the inherent indefiniteness of FC any. The HornGiannakidou approach founders because it sometimes taps into the covert generic operator and sometimes into the added feature of variation/ indiscriminacy, without articulating the conditions under which one or the other comes into play. An account that imputes the universality of FC any to a single source, be it a universal quantifier or implicatures associated with it, we have seen, has a better chance at accounting for the array of facts discussed here.

\section{Conclusion}

There are three main points that I have made in this paper. I have shown that the quantification associated with FC any is universal, whether we choose to 
represent that as a quantifier or by positing special interpretational possibilities for an indefinite. I have also demonstrated that while FC any is semantically compatible with indefinites, it is not identical to them. That is, it cannot be analyzed in terms of a Heimian indefinite. This conclusion was backed, on the one hand, by a careful critique of the indefinite-any account, and on the other, by an explicit analysis of supplementary/numeral any. The scrutiny of arguments for the indefinite-any view revealed several empirical inadequacies and brought to light some problematic hidden assumptions. The analysis of supplementary/numeral any given here represents the first attempt to go beyond a descriptive statement of their distribution. Hopefully, it will lead to further investigation of their properties. Finally, I have shown that it is not possible to do away with a constraint like vagueness that makes reference to indeterminacy at the level of the proposition. I would like to conclude this discussion by commenting briefly on the larger cross-linguistic picture of FC items and the role cross-linguistic studies can play in analyses of particular FC items.

Free choiceness, we have seen, can be defined on the basis of distribution as well as on the basis of interpretive flavor. Though the two coincide in many cases, they do not always give clearcut results. Generally speaking, FC items do not occur in episodic sentences. Their natural habitat are modals of possibility, imperatives and habituals. In terms of their interpretation, the guiding intuition is that they yield statements which apply generally to individuals who have the common noun property, not to a given set of such entities. These criteria still leave room for a lot of variation within and across languages. Let us see the implications of variation in this domain for studies of FC, focusing on any in English and tout in French for illustration.

As already established, English has three types of FC items, all of which carry at least an indirect non-cancellable implicature of universality. They all differ, however, with respect to quantificational force and identityoriented modality. Ignorance of identity is sufficient for two of these items, namely, some $N$ or other and ever-free relatives. The two differ, however, in (in)definiteness. While some $N$ or other has not been systematically studied, it clearly has existential force and does not presuppose knowledge about the individuals at issue. Free relatives with ever have been shown to have properties associated with definites and they have specifically been analysed as having supplementary universal force regarding identity (Dayal 1997; von Fintel 2000). Although any statements are also not about specific individuals, we have seen that the modal requirements cannot be satisfied solely on the basis of identity. As far as the quantificational force of any goes, we have seen that its default or basic interpretation is that of a universal. When it is interpreted in a supplementary clause, its universality can be conjoined with existential statements, leading to what I have termed indirect universality. 
Let us turn now to French, where three distinct FC items have also been identified, namely tout, n'importe quel and le moindre. Jayez and Tovena (to appear) demonstrate that tout is a universal and n'importe quel an existential. Tout, however, has a more restricted distribution than any. Of the constructions most of interest to us here, tout occurs in generics and subtrigged episodics. This is as expected. In imperatives with a subtrigged universal reading too, tout is acceptable but in imperatives with an existential bound it is not:
a. Tout chat shasse les souris.
"Any cat hunts mice."
b. Tout étudiante qui a triché a été renvoue
"Any student who cheated was excluded."
c. Punis tout delit.
"Punish any misdemeanor."
d. *Prends toute carte. "Pick any card."

It might appear from these data that English any is, after all, ambiguous between a universal and an existential while tout is a pure universal. ${ }^{25}$ That is, we would be reverting to the Horn-Giannakidou view for cases like (53d). This move, though tempting, would not be justified. We have already seen evidence in section 4.1 against an ambiguity hypothesis for English. It is worth noting that Jayez and Tovena (to appear), who bring out these differences between any and tout, also stop short of positing such ambiguity for any. I would like to suggest that the source of the difference between these two universal FC items lies elsewhere and I will suggest one possible locus of inquiry.

Consider the fact that tout is not acceptable in supplementary constructions. (54a) would have a representation such as (54b), following the analysis of supplementary constructions proposed in this paper for English FC any: ${ }^{26}$
a. *Prends une carte, (ca pourrait être) toute carte "Pick a card, (it could be) any card."
b. $\mathrm{IMP}_{\mathrm{i}} \exists \mathrm{x}[\operatorname{card}(\mathrm{x}) \&$ pick-up(you, $\mathrm{x}) \&$ antecedent clause $\forall \mathrm{y}\left[\operatorname{card}(\mathrm{y}) \rightarrow \diamond_{\mathrm{i}}\right.$ pick-up(you,y)]] supplementary clause

We can see that this analysis only works if the supplementary FC item is able to take scope over the modal. The opposite order contradicts the existential bound implied by the antecedent sentence. I believe it might be worth 
exploring whether a difference in scope possibilities is at the root of the distributional difference between the two FC items, rather than their quantificational profile.

Although no detailed analysis of the scope properties of any has been done, it has been observed by many that any differs from the universal quantifier every in this respect. ${ }^{27}$ For example, (55a) but not (55b) is acceptable if uttered in a context where there can be only one winner in the race. And every in imperatives is not compatible with an existential reading. That is, a sentence like (55c), though grammatical, cannot be uttered in magic trick contexts where only one card has to be picked:

(55) a. At this point in the race, any horse could win.

b. \#At this point in the race, every horse could win.

c. \#Pick every card.

We can take these facts as indicating that any supports a scope order where the universal scopes over the modal while every supports only the opposite order. Now, turning our attention back to tout, we see that it behaves like every rather than any with respect to scope effects. In the context of interest, the following is unacceptable: ${ }^{28}$

(56) \#A ce point des choses, tout cheval pourrait gagner la course. "At this point, any horse could win the race."

A reasonable conclusion to draw from the data considered here, then, is that tout and any differ in their scope properties not their quantificational force. Their inability or ability to play a supplementary role, I am suggesting, might be due to that difference.

Brief though this foray into a comparison of English and French FC items has been, it has nevertheless highlighted some important considerations as far as crosslinguistic work in this domain is concerned. First of all, the existence of languages such as French, which has lexically distinct universal and existential FC items, make clear the futility of trying to settle on a particular kind of quantification for FC items per se, a point underscored by Jayez and Tovena (to appear). Free choiceness can go along with different types of quantification, universal or indefinite. ${ }^{29}$ The quantification for each FC item in a language must therefore be settled independently and the common core that applies across the board to all FC items extracted from that. At present, this common element seems to be a non-cancellable implicature that the core predication applies in some sense to all possible members of the set denoted by the common noun. Furthermore, languages like French with separate lexical items open up possibilities for investigations in languages like 
English where a single item seems to display mixed properties. However, our discussion here has also demonstrated the need for caution in applying insights from one language to another. FC items across languages, even when they agree on quantification and modality, may well show an overlap in behavior rather than complete uniformity. The task then turns into a search for other factors to explain the observed differences. If that search is successful, we can say we have made further headway in understanding the range of possible cross-linguistic variation. Ultimately, however, what we learn from one language or one set of languages is only a guide in deciphering the situation in another language or another set of languages. Hypotheses about each FC item have to be argued for on the basis of language internal evidence, providing detailed analyses of particular constructions for testing out the hypotheses.

The investigation into the quantificational properties of FC any presented here has tried to follow these guidelines. The possibility of an indefinite FC item was inspired by work on languages such as French, Italian and German as well as by English data that did not fit neatly into the paradigm of universal-any. Based on a particular analysis of these constructions, as well as on arguments from previous work, I have claimed that FC any in English always encodes universality though this universality is of a kind that can be conjoined with independently established existential statements.

\footnotetext{
Notes

${ }^{1}$ This paper owes much to the lively discussions in the Seminar on Free Choice (Rutgers University, Spring '04). I also thank the colloquia audiences at University of Maryland and CUNY as well as an anonymous reviewer of this paper for useful feedback. Finally, thanks to Yael Sharvit for helpful discussion. Many thanks to Daniel Altshuler for editorial assistance. I remain responsible for all errors and omissions.

${ }^{2}$ One caveat noted in Dayal (1998), attributed to Barbara Partee, has to do with preposed adverbs such as rarely and seldom which do allow for quantificational readings: Rarely/seldom is any lion majestic. Given the nature of the adverbs involved, this may well be an instance of negative polarity any.

${ }^{3}$ An anonymous reviewer objects on the grounds that we end up with ungrammaticality as opposed to presupposition failure in cases like (4a) and (7). The point is well taken but there is a plausible explanation for this. When a statement $q$ with presupposition $\mathrm{p}$ is uttered in a context where $p$ is not true, we have a case of presupposition failure. That is, had the context been one where $\mathrm{p}$ could have been true, q would have been considered felicitous and evaluated as true or false. The presupposition of a statement q' with an any phrase in it, I am claiming, is that the predication could in principle hold of the set any quantifies over. Since no context can support such a presupposition, without the help of overt or covert subtrigging, the resulting intuition is stronger than that of infelicity.
} 
${ }^{4}$ Giannakidou (2001: 727) gives an example (cf. her 183) showing that explicit domain narrowing is not sufficient to license any and notes this to be a problem for Dayal's account. However, she misses the fact that her use of partitive any in an episodic statement violates vagueness. So, in fact, the unacceptable sentence is ruled out on the universal-any account, contrary to Giannakidou's understanding of that account. I am not giving an explicit demonstration to show this, since the explanation for her example would be fully parallel to the explanations for (10b), (13b) and (15b) provided here.

${ }^{5}$ I owe the inclusion of this section to a spirited defense of the indefinite-any position put forth by the anonymous reviewer of this paper. It forced me to articulate the weaknesses in the indefinite-any view in some detail.

${ }^{6}$ Jayez and Tovena (2004: 41), while agreeing with the core data, present the following as a possible counterexample: Any lion is normally able to run one kilometer, but, on this planet they don't move very easily. Note that this example does not involve a quantificational reading. The first sentence makes a claim about capabilities of all lions under normal conditions. The second sentence can only be intepreted if we take the conditions on this planet to be abnormal. If the second set of lions do not run a kilometer because of the abnormal conditions on this planet, they do not falsify the claim in the first sentence. This is therefore not a valid counterexample to the claim that adverbs of quantification do not bind any phrases. Note that the example does not go through if normally is replaced with often or usually.

${ }^{7}$ Giannakidou later changes the variation to a conversational implicature that can be cancelled. I am not sure how that move impacts on the discussion of Q-adverbs.

${ }^{8}$ In an earlier section Giannakidou (2001: 701-703) has a mystifying discussion about Qadverbs. She says, "Dayal (1998:438) claims that any, unlike regular existentials, cannot be construed with Q-adverbs in generic contexts, but this is not true of FCIs, or of any, for that matter". In that very same section, however, she goes on to say "to be fair, the interpretation of FCIs and any in generic contexts of this type differs from that of regular indefinites in one particular respect: an example [with $\mathrm{FCI}$ ] seems to convey a generalization about all [N], whereas the sentence with a regular indefinite seems to be about most [N]". I leave it to the reader to judge the validity of the first statement for themselves. Since the proffered explanation obviously takes the second statement as its goal, and that is the statement that is consistent with native speaker judgments, I have used that in my evaluation of the proposal here and set aside the first statement.

${ }^{9}$ Giannakidou (2201: 716) also notes in her discussion of this phenomenon that statements with the regular universal differ from those with any in quantifying over an extensional set. This point, however, is orthogonal to the issue under discussion in that subsection.

Furthermore, the difference in quantificational domains between any and a regular universal is already part of the universal-any account, so the objection seems to be based on a partial reading of that account.

${ }^{10}$ It is not clear to me what role the intensionality of any plays in Giannakidou's account. As far as I can tell, it is meant to prevent any from occurring in episodic statements. Since subtrigged cases are treated by Giannakidou as forcing a conditional structure due to iterativity, the postulated difference in intensionality cannot be used to argue away this problem.

${ }^{11}$ In the account in Dayal (1998) iterativity is considered a sufficient condition for any because it indirectly makes it possible for vagueness to be satisfied. If the number of events is openended it follows that the set of individuals who participate in those events need not be 
contextually fixed. Jayez and Tovena (to appear), however, note cases where any is not licensed even when an iterative interpretation is available.

${ }^{12}$ For Saeboe (2001), a FC item has two components. One component is an intensionalizer, the other a universal quantifier. Any occurs with modals, in this view, because modals serve to undo the intensionality introduced by any. They thereby pave the way for the universal quantifier, which is assumed to be defined only for extensional domains. This account, of course, cannot discriminate between necessity and possibility whose contribution to types is the same. If one can extensionalize, so can the other.

${ }^{13}$ I am grateful to Bob Fiengo and Karuna Srivastav for first drawing my attention to supplementary and numeral any.

${ }^{14}$ The anonymous reviewer notes that the unacceptability of (33) is to be expected, given that dream is veridical. The point, however, is that verbs like look-for and wait, whether veridical or not, reject ordinary any but accept numeral/supplementary any. Thus, no matter how the definition of (non)veridicality is tweaked it will not be able to explain this contrast.

${ }^{15}$ Note that under this view quantifiers are ruled out as potential antecedents. There is in essence an E-type pronoun in the supplementary clause that requires an indefinite in the antecedent clause. Thus, the restriction on the type of antecedents noted in Jennings (1994) is expected.

${ }^{16}$ Chierchia (2004) is not able to derive the absence of the upper bound in necessity statements. See section 4.3 for further discussion of his proposal.

${ }^{17}$ Von Fintel (2000) argues in favor of treating the contribution of -ever as a presupposition rather than part of the assertion.

${ }^{18}$ I assume that a context of ignorance will satisfy the presupposition of any (cf. (16) above) in Giannakidou's account but neither Horn nor Giannakidou clarify how differences between FC any and FC free relatives are to be derived. Therefore, it is hard for me to make this statement with greater assurance.

${ }^{19}$ The following examples were provided by the reviewer of this paper as evidence against the view that dream/imagine do not support purpose clauses:

i. John dreamt of a woman to fall in love with.

ii. John imagined a world to live peacefully in.

It is not clear to me that these cases are parallel to the ones under discussion. Note that we cannot render these examples using a so that phrase: John dreamt of a woman so that he could fall in love with her or John imagined a world so that he could live peacefully in it seem quite odd. They certainly do not have the same meanings as (i) and (ii), where the so-called purpose phrases seem to provide a restriction on the objects at issue: a woman who is worth falling in love with or a world of the sort one could live peacefully in. John was looking for a policeman so that he could report the crime, on the other hand, is equivalent to (45a) and John wanted a book so that he could read it to (45b). It appears that this is the type of purpose clause that supplementary any is sensitive to.

${ }^{20}$ Jayez and Tovena (2004) acknowledge the existence of universal FCI and existential FCI in French and hence cross-linguistically. They demonstrate that English any, however, has mixed properties and remain agnostic about its quantificational character. 
${ }^{21}$ I set aside here the issue of quantificational domain and vagueness, which would obviously have to be included to derive the empirical coverage of the universal-any account developed in the earlier sections.

${ }^{22}$ Recall that the notion of strengthening used by Kadmon and Landman (1993) was meant to rule out existential any in affirmative contexts. This marks a shift in the analysis of FC items as indefinites inducing widening.

${ }^{23}$ As mentioned earlier, Chierchia's analysis of indefinite FC items cannot derive the fact that commands do not place restrictions on size while permissions do (cf. (38)).

${ }^{24}$ Chierchia shows that the absence of quantificational variability with adverbs follows from his account. He also demonstrates how the explanation for the subtrigging effect given in Dayal (1998) can be incorporated. As far as I can tell, the differential behavior of possibility and necessity with respect to partitive any, for which the vagueness requirement was critical, is not captured.

${ }^{25}$ Under this view, English would have one more FC item, namely a non-identity oriented indefinite.

${ }^{26}$ Many thanks to Viviane Deprez for help with these data.

${ }^{27}$ Giannakidou (2001: 694-697) points to cases where any does not take scope over a ccommanding indefinite. This, according to her, argues for an account of any as an indefinite with a presupposition of variation. Note, however, that in sentences like (55a), any cannot be treated as an existential with scope under the modal. This highlights the need for a more rigorous study of the scope properties of this item.

${ }^{28}$ Viviane Deprez (p.c.) notes that the sentence is not completely out, just rather odd. She further notes that there is some improvement if an adjective is added: tout bon cheval, "any good horse". She also notes that there are other contexts where tout seems to take scope over a modal: A ce point des choses, tout pourrait arriver, "At this point, anything could happen".

${ }^{29}$ Free choice is also, of course, compatible with definiteness, as argued for English free relatives (Jacobson 1995; Dayal 1995b and 1997; and von Fintel 2000) as well as for Hindi correlatives (Dayal 1995b).

\section{References}

Chierchia, Gennaro. 2004. "Broaden Your Views: Implicatures of Domain Widening" ms. University of Milan-Bicocca.

Dayal, Veneeta. 1995a. "Licensing Any in Non-negative/Non-modal Contexts" Proceedings of SALT V.

Dayal, Veneeta. 1995b. "Quantification in Correlatives". Quantification in Natural Language ed. by Emmon Bach, Eloise Jelinek, Angelika Kratzer and Barbara Partee, 179-205. Dordrecht: Kluwer.

Dayal, Veneeta. 1997. "Free Relatives and -ever: Identity and Free Choice Readings" Proceedings of SALT VII.

Dayal, Veneeta. 1998. "Any as Inherently Modal" Linguistics and Philosophy 21. 433-76. 
von Fintel, Kai. 2000. "Whatever" Proceedings of SALT X.

Giannakidou, Anastasia. 2001. "Affective Dependencies" Linguistics and Philosophy 22. 367-421.

Giannakidou, Anastasia. 2001. "The Meaning of FC" Linguistics and Philosophy 24. 659-735.

Horn, Lawrence R. 2001. "Any and -ever: Free Choice and Free Relatives". Proceedingsof the $15^{\text {th }}$ Annual Conference of the Israeli Association for Theoretical Linguistics 71-111.

Horn, Lawrence. R. 2003. "Airport'86 Revisited: Toward a unified indefinite any" ms. Yale University.

Jacobson, Pauline. 1995. "On the Quantificational Force of English Free Relatives". Quantification in Natural Language ed. by Emmon Bach, Eloise Jelinek, Angelika Kratzer and Barbara Partee. 451-86. Dordrecht: Kluwer.

Jayez, Jacques and Lucia Tovena. 2004. "Free Choiceness and NonIndividuation" Linguistics and Philosophy.

Jennings, Raymond E. 1994. The Genealogy of Disjunction. New York: Oxford University Press.

Kadmon, Nirit and Fred Landman. 1993. "Any" Linguistics and Philosophy 16. 353-422.

Kratzer, A. and J. Shimoyama: 2002, "Indeterminate Pronouns: The View from

Japanese”, Paper presented at the $3^{\text {rd }}$ Tokyo Conference on Psycholinguistics.

LeGrand, Jean E. 1975. Or and Any: The Semantics and Syntax of Two Logical Operators. Doctoral dissertation, University of Chicago.

Quer, Josep. 1998. Mood at the Interface. Doctoral dissertation, University of Utrecht. The Hague: Holland Academic Graphics.

Sadler, L. and D.J. Arnold: 1994, 'Prenominal Adjectives and the Phrasal/Lexical Distinction', Journal of Linguistics 30. 187-226.

Saeboe, Kjell Johan. 2001. "The Semantics of Scandinavian Free Choice Items" Linguistics and Philosophy 24. 737-787.

Vendler, Zeno. 1967. Linguistics in Philosophy. Ithaca: Cornell University Press.

Vlachou, Evangelia. 2003. "Why should not little John Forget his doll, anywhere he goes?" ms.University of Utrecht. 
Zepter, Alex. 2003. "How to be Universal when you are Existential: Entailment along a Scale" Journal of Semantics 20. 193-237.

Zimmermann, Thomas Ede. "On the Proper Treatment of Opacity in Certain Verbs" Linguistics and Philosophy. 149-179. 